ARCHIVO ESPAÑOL DE ARTE, LXXXIV, 335

JULIO-SEPTIEMBRE 2011, pp. 197-210

ISSN: 0004-0428

\title{
ALGUNOS APUNTES SOBRE LA NATIVIDAD DE FEDERICO BAROCCI EN EL MUSEO DEL PRADO
}

\author{
GONZALO REDÍN \\ Investigador contratado Programa Ramón y Cajal. Universidad de Alcalá
}

\begin{abstract}
El texto propone una lectura de la Natividad de Barocci en el Museo del Prado como una alegoría eucarística, por la presencia de las espigas de trigo, de grano y de pan en ella. La pintura se estudia en relación a sus dibujos preparatorios, uno de los cuales, conservado en el Louvre, no se ha tenido en cuenta hasta la fecha, y analiza el modo de trabajo de Barocci, que debió modelar una escultura inspirada en la figura de Cristo en la Oración del huerto de Correggio para estudiar la posición de María.
\end{abstract}

Palabras clave: Barocci; Correggio; Brandani; Natividad; Eucaristía.

\section{SOME NOTES ON FEDERICO BAROCCI'S NATIVITY IN THE PRADO MUSEUM}

This text proposes a reading of Barocci's Nativity in the Prado Museum as a Eucharistic allegory, due to the presence of ears of wheat, grain, and bread in the composition. The painting is studied in connection with its preparatory drawings (one of which, in the Louvre, has not been considered to date), and analyses Barocci's working method: he must have modelled a sculpture inspired by the figure of Christ in Correggio's Agony in the Garden in order to study the position of Mary.

Key words: Barocci; Correggio; Brandani; Nativity; Eucharist.

En 1597 el duque de Urbino, Francesco Maria della Rovere II, pagaba a Federico Barocci el cuadro de la Natividad que ahora se encuentra en el Museo del Prado (fig. 1). En septiembre de 1604 el duque de Sesa, atendiendo a los deseos de la reina Margarita de Austria, preguntaba al embajador del duque de Urbino en España, Bernardo Maschi, noticias sobre Barocci, en lo que era prácticamente una orden velada para obtener una obra del pintor, toda vez que la política de supervivencia del estado de los della Rovere, amenazado por las aspiraciones del papado, dependía de la protección española ${ }^{1}$. Un año después, el propio duque, sabedor de que los lentísimos tiempos de ejecución de Barocci hacían imposible que éste aceptara un nuevo encargo, decidió enviar a la reina el único cuadro del pintor que quedaba en su colección, una Natividad de la que, como afirma en una carta a Maschi: "se privaría a mala gana" y sólo si el cuadro "fuera reconoci-

${ }^{1}$ Bellori, G. P., Le vite de' pittori, scultori et architetti moderni, Roma, 1672, p. 194. Gronau, G., Documenti artistici urbinati, Florencia, Sansoni, 1936, pp. 173-175. 
do y estimado cuanto merece"2. Sin duda tenía presente la réplica de la Vocación de San Pedro de Barocci que Felipe II había solicitado para la decoración de El Escorial, donde se encuentra, y que llegó a Madrid en 1588. Una obra que, en palabras del embajador de Urbino, gustó en general mucho, aunque "los malhablados y los envidiosos no han dejado de criticarle que el Cristo tenía que ser de estatura mayor y que San Andrés habría sido más proporcionado con una pierna más delgada" 3 . Afortunadamente la Natividad fue del agrado de la reina Margarita que, como buena entendida, se tomó la molestia de colocar la tela de manera que la luz fuera lo más favorecedora posible y la consideró obra muy buena, además de "muy alegre y muy devota"4, característica, esta última, típica de la producción de Barocci, que en la corte española, todavía heredera del gusto de Felipe II, había de ser más importante que la del valor exclusivamente estético de la pintura.

Una réplica del cuadro realizada en el taller de Barocci, fue encargada, todavía en vida del pintor, por Federico Borromeo ${ }^{5}$. No sorprende, desde luego, que el arzobispo de Milán quisiera tener una obra suya. Como discípulo de Filippo Neri e importante benefactor de los oratorianos de Chiesa Nuova, contribuyó a que el pintor de Urbino recibiera hasta cuatro comisiones para esta iglesia, y pretendió otras cuatro para el Duomo de Milán ${ }^{6}$. La pintura es citada en 1607 en su codicilio testamentario y tras la muerte del prelado en 1631, fue a parar a la Pinacoteca Ambrosiana $^{7}$. Aunque el original del Prado fue realizado para Francesco Maria della Rovere, no cabe duda de que la obra de Barocci se adecuaba como pocas al ideal de la "Cristiana Leticia" propugnado por Filippo Neri, que insistía en retornar a la sencillez de las fuentes evangélicas para acercarse a los fieles más ignorantes ${ }^{8}$. No en vano, según refieren varias fuentes, el santo estimaba mucho la Visitación que Barocci pintó para la capilla Pizzomiglio de Chiesa Nuova, ante la que se retiraba a menudo a orar en la intimidad 9 . Si bien es cierto que Federico Borromeo no menciona directamente la pintura de Barocci en su Musaeum, en el que incluye las obras más importan-

2 Gronau, 1936, 174. Ya en 1590 Diego Fernández Cabrera-Bobadilla, III Conde de Chinchón, secretario de Felipe II, solicitó al Duque de Urbino una pintura de Barocci para el convento de Clarisas de Chinchón; el duque consideró imposible atender la petición, por la lentitud del pintor y el mérito escaso de sus discípulos, pero una obra de alguno de ellos llegó a Chinchón en 1608, y aunque sus dimensiones eran inadecuadas, debió gustar, porque el conde pidió inmediatamente una Pentecostés. G. SEMEnZA, "La scuola baroccesca nelle note di spese di Francesco Maria II della Rovere" en A. Marchi, "Alessandro Vitali" en Ambrosini Massari, A. M. Cellini, M., Nel segno di Barocci: allievi e seguaci tra Marche, Umbria, Siena, Milán, Motta, 2005, p. 38.

3 Emiliani, A., Federico Barocci (Urbino, 1535-1612). II, Ancona, 2008, p. 188.

${ }^{4}$ Gronau, 1936, p. 174. La pintura estaba en 1793 en la Casita del Príncipe, Carlos IV, en El Escorial. JordáN Hurríes de la Colina, J. J., La Casita del Príncipe en El Escorial, 2009, p. 26.

5 Últimamente Verstegen señala que esta obra puede ser el Presepe encargado por el capítulo de la catedral de Milán. Sin embargo, sus pequeñas dimensiones no parecen muy adecuadas para este edificio y las cartas escritas por Borromeo a Fra Damiano en Urbino y al propio Barocci no especifican de que tema se trata. Por otro lado, no se entiende que, en el caso de que la obra fuera original, no se incluya en el De Pictura Sacra de Borromeo. Verstegen, I., "Federico Barocci, Federico Borromeo, and the Oratorian Orbit", Renaissance Quarterly, 56, 2003, pp. 56-87 (71-72). Bonomelli, M., "Federico Barocci e la committenza milanese della Fabrica del duomo", Libri \& documenti. Archivio Storico Civico e Biblioteca Trivulziana, 19, 1993, 1, pp. 18-25. Olsen, H., F. Barocci. A critical study in Italian Cinquecento painting, Estocolmo, Almqvist \& Wiksell, 1955, pp. 153-154. EMILIANI, 2008, p. 206.

6 Vestegen, 2003, p. 86. Olsen, 1955, p. 56.

7 Jones, P. M., Federico Borromeo and the Ambrosiana: art patronage and reform in seventeenth-century Milan, Cambridge, Cambridge Univ. Press, 1993, pp. 69 y ss. Emiliani, 2008, p. 320.

${ }^{8}$ EmiLiani, 2008, pp. 32-33 y 43.

${ }^{9}$ En la capilla y frente al cuadro, según refieren algunos testigos en su proceso de santificación, realizó varias curaciones. BACCI, P. G., La vita di San Filippo Neri, Roma, 1622, p. 132. Olsen, 1955, pp. 25 y 28. InCisa Della Rocchetta, G. y Vian., N., II Primo Processo per San Filippo Neri, Vaticano, 1957-1963, I, pp. 273, 330, 337, 340; II, pp. 113, 125. VERSTEGEN, 2003, p. 65. 


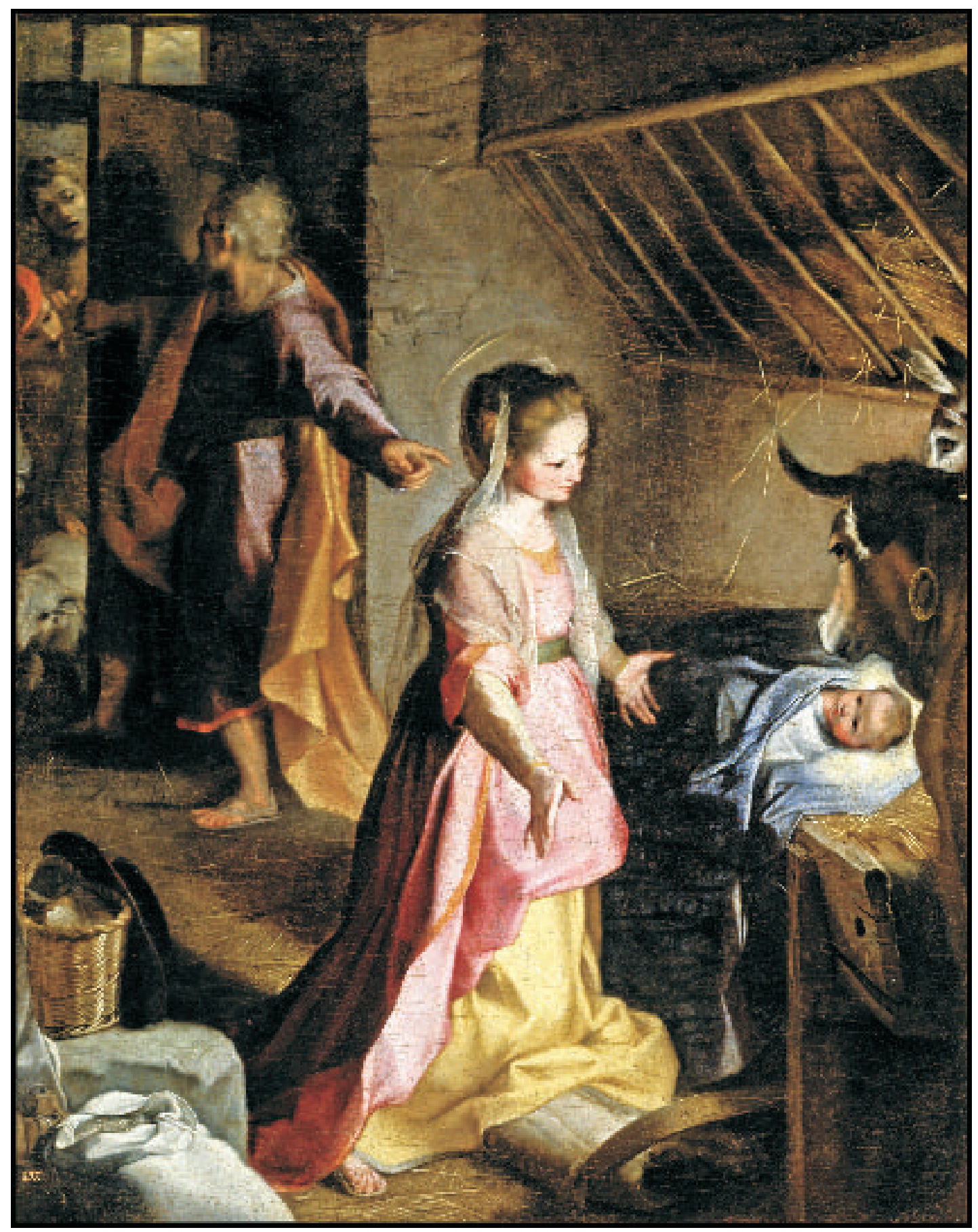

Fig. 1. Federico Barocci. Natividad. Museo Nacional del Prado.

Arch. esp. arte, LXXXIv, 335, JULIO-SEPTIEMBRE 2011, 197-210, ISSN: 0004-0428 
tes de su colección, ni en De Pictura Sacra, en este tratado recoge los temas más apropiados para la edificación de los fieles, señala como habían de representarse correctamente y hace especial hincapié en la importancia de la claridad narrativa y en la expresión de los affetti, dedicando notable atención al tema de la Natividad ${ }^{10}$. En su opinión, la lectura de este episodio evangélico estaba siempre penalizada por el excesivo artificio de los pintores, que representaban a los pastores con unos cuerpos tan atléticos y llamativos que el fiel no atendía como era debido al motivo principal del cuadro. En sus palabras, las actitudes y poses de los pastores no despertaban "una chispa de piedad"11. Todo lo contrario, desde luego, a lo que ocurre en la pintura de Barocci, en la que todos los recursos formales se emplean para que el espectador concentre su atención en el episodio principal, la adoración de la Virgen al niño Jesús. La cabeza del buey, en primer plano, y las figuras de José, al fondo, con el brazo extendido hacia el niño, y de los pastores, que también dirigen su mirada al mismo, conducen la mirada del fiel a Jesús. Por otra parte, la luz irreal que emana de Cristo Niño y que ilumina a María, se confronta con la dulce y doméstica penumbra del resto de la escena, que contrasta con el encendido colorido de las vestimentas de María y su hijo. Se trata, pues, de la obra de un pintor "vago e divoto", palabras de Baglione que, como ha demostrado ejemplarmente Lingo en su monografía sobre Barocci, es capaz de atender a las exigencias devocionales sin menoscabar por ello los valores estéticos de la pintura ${ }^{12}$.

Como es sabido, el nacimiento de Cristo es narrado de manera muy sumaria en el Evangelio de San Mateo y, con más detenimiento, en el de San Lucas, donde se dice que después de buscar infructuosamente un albergue, María tuvo que acostar al niño en un pesebre. Como tantas veces, la falta de información alimentó la imaginación popular, de manera que los evangelios apócrifos añadieron algunos personajes, como la matrona crédula y la incrédula o la aparición de los pastores que vienen a adorar al Niño, que Barocci sitúa inteligentemente en último plano y cuya presencia, ciertamente, no distrae la atención del fiel ${ }^{13}$. De esta manera, Barocci pinta un cuadro que representa antes a María adorando al niño que una Adoración de los pastores. La acelerada perspectiva de "sotto in su", marcada por la diagonal (de inevitable memoria correggesca) que recorre todo el cuadro desde la cabeza del buey hasta llegar a los pastores (y que es subrayada por la extensión de los brazos de José y de María y reforzada por el haz de luz que recorre detrás de ella, en la misma dirección, el suelo), sitúa al fiel como si estuviera presente arrodillado junto al animal, y muy cerca de la madre y el niño, haciéndole espectador directo y partícipe de la íntima relación que se establece entre ambos.

Pero la pintura no es sólo una representación del nacimiento de Jesús. La presencia de la cesta con pan en primer plano, acompañada por un saco de grano y, esto es más llamativo, por las espigas de trigo que podemos ver entre María y su hijo, sugieren una posible alusión eucarística. Alusión que podría ser literal si consideramos que el cruce de las dos espigas entre las cabezas de madre e hijo es demasiado rebuscado para no ser premeditado, porque forma una cruz, tal y como sucede, en opinión de Lingo, en el caso de la paja y los clavos situados en el inferior del Entierro de Cristo de Barocci en Senigallia ${ }^{14}$. En el Evangelio de San Juan, Cristo se identifica con el pan: "Yo soy el pan, descendido del cielo" (Juan, VI, 51), y con el grano de trigo: "De cierto os digo, que si el grano de trigo no cae en la tierra y muere, queda solo; pero si muere, lleva mucho fruto" (Juan, XII, 24). El grano de trigo se rompe en la tierra para tener una vida nueva, debe morir para germinar. Al igual que el grano muerto produce la espiga repleta de fruto, la muerte de Cristo, con su posterior resurrección, da lugar a la iglesia. De esta manera, la

\footnotetext{
10 JONES, 1993, pp. 32-34 y 114.

11 Idem, pp. 113-114

12 Lingo, S., Federico Barocci. Allure and Devotion in Late Renaissance Painting, Londres, 2007.

13 ReAu, L., Iconographie de l'art chrétien, II, p. 123, París, 1956, pp. 221-229.

14 LiNGO, 2007, p. 108.
} 
presencia del trigo, del grano y del pan, sirve para establecer un paralelismo entre la Natividad, que representa el momento de la encarnación de Jesús, del Verbo hecho hombre, y la Eucaristía, el momento en que la humanidad de Cristo se manifiesta definitivamente en su muerte en la cruz. No hay que olvidar que el pan aparece, acompañado por un recipiente que cabe suponer contiene vino, en la parte más baja de la composición, casi sobre el altar donde se celebra la eucaristía, en otras obras de Barocci como el Descanso en la huida a Egipto (Pinacoteca vaticana), el Martirio de San Vital (Brera) o la Visitación (Chiesa Nuova) ${ }^{15}$.

Por otra parte, la cesta de pan, el trigo y el grano pueden ser interpretados como una alusión a Belén, el lugar del nacimiento señalado por los evangelistas Lucas y Mateo, que en hebreo significa casa del pan. En la homilía que San Gregorio dedica a la Navidad afirma: "Qui bene etiam in Bethlehem nascitur: Bethlehem quippe domus panis interpretatur. Ipse namque est qui ait. Ego sumus panis vivus qui de caelo descendi" 16 , y este significado tiene la presencia del trigo en la Natividad de Hugo Van der Goes en los Uffizi. En este contexto de alusiones eucarísticas, no parece casual que por la puerta que San José abre a los estupefactos pastores entre un carnero, y no el cordero que habitualmente simboliza a Cristo. La cornamenta del carnero era vista como un "jeroglífico" de la cruz, y el animal es empleado también como imagen sacrificial de Cristo, como sucede, por ejemplo, en las pinturas de San Juan Bautista de Caravaggio de la Pinacoteca Capitolina y de la Galleria Borghese. Así aparece en las representaciones paleocristianas, tan caras a los oratorianos por su sencillez, del Sacrificio de Isaac, donde se incluye un carnero para evidenciar que el episodio del Antiguo Testamento prefigura el sacrificio de Cristo en la cruz, la Eucaristía, mientras que el símbolo del Aries acompaña no pocas veces el nombre de Jesucristo ${ }^{17}$. La inclusión del carnero (Agnus carnarius, esto es, cordero de carne, destinado sólo a servir de alimento), puede aludir también a Belén, que en árabe significa casa de la carne, y a la propia encarnación de Jesús en el momento de su nacimiento en Belén y en la crucifixión. Por lo demás, Cristo afirma: "Yo soy el pan vivo que descendió del cielo; si alguno come de este pan, vivirá para siempre. El pan que yo daré por la vida del mundo es mi carne" (San Juan, VI, 51-52), una carne a la que puede referirse directamente el animal que entra en la habitación.

No parece que se pueda indicar una fuente iconográfica concreta para la escena, que deriva, desde luego, de las Revelaciones de Santa Brígida. María está de rodillas, adorando al niño, que es fuente de luz, y el hecho sucede en Belén, como parecen indicar las diferentes alusiones al pan, sin embargo, el nacimiento no tiene lugar en una cueva, como refiere la santa ${ }^{18}$. Resulta difícil identificar los objetos a los pies de la Virgen. ¿Un haz de leña recogido en una tela blanca, de aspecto algodonoso? ¿Una especie de yunta dorada decorada con algunos signos que parecen sólo ornamentales? Probablemente la tela blanca enrollada bajo María sea alguno de los paños de lino que, según la visión de Santa Brígida, la virgen empleó para arropar al Niño ${ }^{19}$.

Pseudo-Buenaventura señala que el buey y el asno se acercaron al recién nacido para calentarle con su aliento, y así se les figura muy a menudo, casi siempre reclinados en señal de ado-

\footnotetext{
${ }^{15}$ LingO, 2007, pp. 169-170.

16 Migne, P. L., Patrologia Latina, LXXVI, 1104. PAnOFKsy, E., Early Netherlandish Painting: Its Origins and Character, Cambridge, 1953, pp. 333 y 500, n. 333. Lane, B. G., "Ecce Panis Angelorum: The Manger as Altar in Hugo's Berlin Nativity", The Art Bulletin, 57, 1975, pp. 476-486, n. 30.

17 Calvesi, M., "Letture iconologiche del Caravaggio", en CinotTi, M., Novità sul Caravaggio, saggi e contributi, 1975, Cinisello Balsamo, pp. 75-102. Calvesi, M., Caravaggio, Florencia, 1986, p. 17.

18 Santa Brígida de Suecia, Celestiales revelaciones, Madrid, 1901, libro 7, cap. XII.

19 A su izquierda, dos estribos de madera parecen referirse a la llegada de María a Belén a lomos del asno.

Santa Brígida de Suecia, 1901, libro 7, cap. XII. O, más difícilmente, el cojín de lana que, según se refiere en las Meditaciones de Pseudo-Buenaventura, José colocó junto al pesebre para que su mujer descansara. Pseudo-Buenaventura, "Meditationes Passionis Iesu Christi" en Amorós, L., Aperribay, B., Oromi, M., Obras de San Buenaventura, II, BAC, Madrid, 1937.
} 
ración y respeto. Por eso llama mucho la atención que ambos animales se encuentren de pie, y más aún que el buey aparezca en un primerísimo plano, por delante de María y Jesús y con su cabeza casi a la altura de la Virgen. Algo que podría resultar indecoroso, pero que Barocci resuelve dignificando a los animales, estudiados del natural (porque su representación convencional, anecdótica, aquí no basta, se trata de dos personajes más), con una actitud solemne que les muestra conscientes de la importancia del hecho que presencian. Es posible que la posición destacada del buey y el asno aluda a los judíos y los gentiles ${ }^{20}$, pero teniendo en cuenta la estrecha filiación de Barocci a lo largo de toda su vida con las diferentes órdenes franciscanas, con las que fue muy generoso en su testamento, en el que pidió ser sepultado en San Francesco de Urbino $^{21}$, cabe pensar que compartía la actitud del santo hacia los animales, y que por ello los humanizó en sus pinturas. Sabido es que San Francisco creó el primer pesebre en Greccio para poder "contemplar con mis ojos corporales cómo era aquello de estar recostado en un pesebre y dormir sobre las pajas entre un buey y un asno", y que llegó a celebrar misa con la presencia de los dos animales mansos, que estaban entre sus preferidos ${ }^{22}$. San Francisco consideraba la Navidad como la fiesta más importante, porque la salvación del hombre comenzaba ya con el nacimiento de Cristo, y quería que ese día los ricos dieran de comer en abundancia a los pobres y hambrientos, y que los bueyes y los asnos tuvieran más pienso y hierba de lo habitual ${ }^{23}$.

La obra llama la atención, ante todo, por el extraordinario y equilibrado contraste logrado entre la representación casi realista del establo y la idealización de las figuras, absolutamente angélicas, del Niño Jesús y de María, cuyo rostro replica el de la Virgen esculpida por Federico Brandani en el Nacimiento del Oratorio de San Giuseppe de Urbino (fig. 2). Así, la convincente figuración de todos los accidentes de la madera del pesebre o de los desconchados del muro, resulta relativamente chocante frente a la extrema estilización de la figura de María, coloreada de manera completamente arbitraria. También la iluminación se emplea a medio camino entre el naturalismo, nótese la extraordinaria proyección de las sombras de los barrotes de madera sobre la parte superior del muro, y el puro artificio. A pesar del matizadísimo efecto de penumbra que domina la obra, Barocci emplea la luz a su antojo, con una intención sólo aparente, y más bien efectista, de estudiar o figurar su disposición en un escenario real. De otro modo, no se explica el "río" de luz que, sin un origen concreto, corre caprichosamente por el pavimento, por detrás de María, entre dos sombras, y que, pese a todo, se integra con naturalidad en la composición.

Y es que la aparente sencillez de esta Natividad es, en realidad, fruto de un arduo trabajo en el que Barocci, partiendo del natural, sigue un proceso enormemente elaborado del que da cuenta Bellori y del que ha quedado testimonio gracias a los muchísimos dibujos que documentan la incansable actividad gráfica del pintor de Urbino ${ }^{24}$. Bellori expone el proceder del pintor de manera interesada, estableciendo unos estadios de concepción y preparación de la pintura que, como ha señalado Turner, no tienen porque ser estrictamente sucesivos, y que son referidos por

${ }^{20}$ Interpretación que tiene su origen en Habacuc y en una exégesis, errónea de Isaías. REAU, II, p. 528.

21 Emiliani, 2008, I, p. 42. Lingo, 2007, pp. 18-24.

22 De Celano, T., Vida segunda de San Francisco (CXXIV, 84), en Guerra, J. A., San Francisco de Asís. Escritos. Biografias. Documentos de la época, BAC 399, Madrid, 1998. De Celano, T., Vida primera de San Francisco (XX, 84), en Guerra, J. A., San Francisco de Asís. Escritos. Biografias. Documentos de la época, BAC 399, Madrid, 1998.

${ }^{23}$ En una ocasión, cuando un hermano le hablaba de que esa Navidad no podrían comer carne porque la fiesta caía en viernes, el santo le rebatió: "Hermano, pecas al llamar día de Venus al día en que nos ha nacido el Niño. Quiero -añadió- que en ese día hasta las paredes coman carne; y ya que no pueden, que a lo menos sean untadas por fuera". Algo con lo que, tal vez, pueda ser relacionado el carnero que entra por la puerta. Idem, Vida segunda, CLI, 200.

${ }^{24}$ BeLlori, 1672, p. 178. 
el teórico como precedente de la praxis clasicista que defiende, exaltando la utilización de dibujos preparatorios, al modo de Rafael o Annibale Carracci, previos a la realización de la obra ${ }^{25}$. Según Bellori, Barocci tomaba a vuela pluma algunos apuntes del natural, en un cuaderno que siempre llevaba consigo.

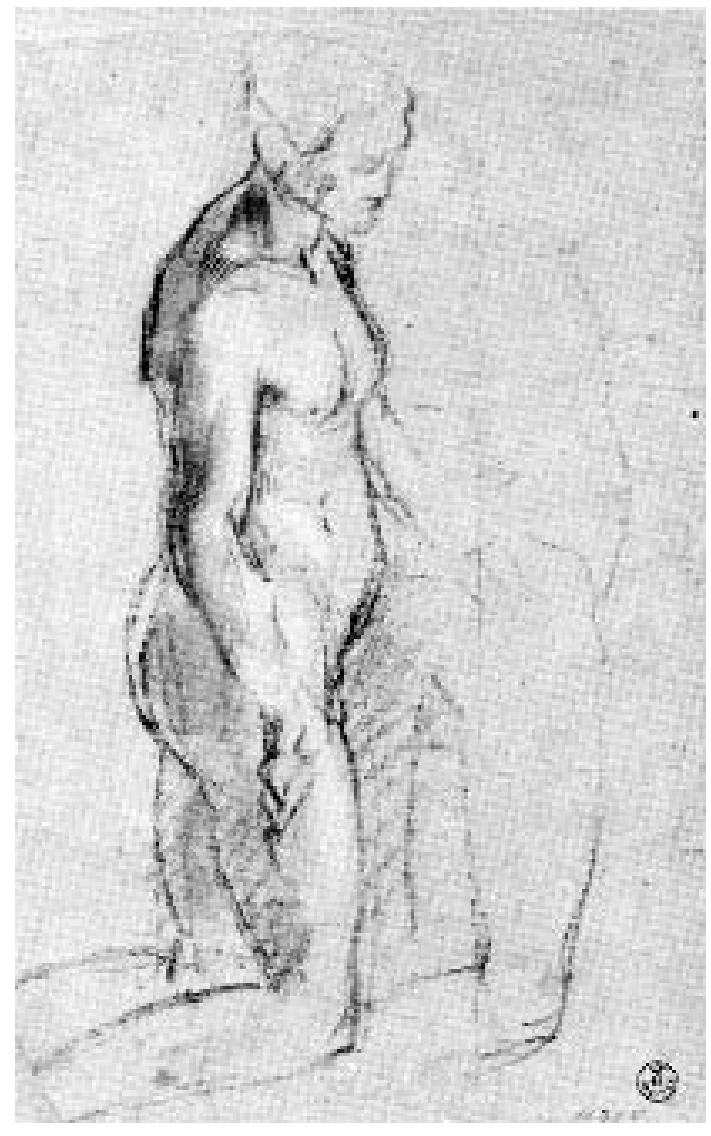

Fig. 3. Barocci. Estudio de figura. Gabinetto Disegni e Stampe degli Uffizi.

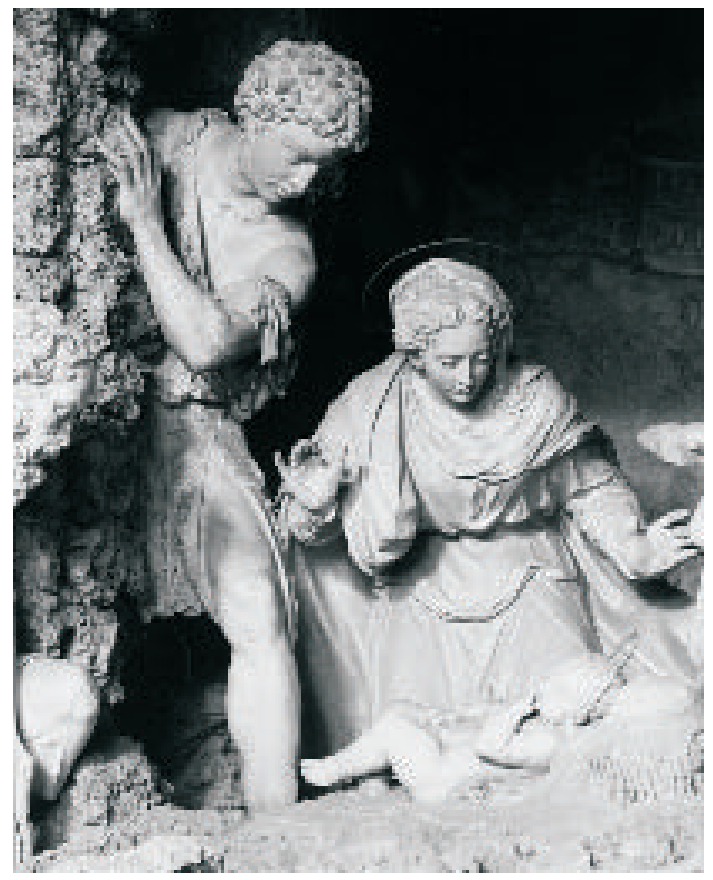

Fig. 2. Federico Brandani. Nacimiento. Oratorio de San Giuseppe. Urbino.

Después de hacer un primer boceto, o "scarpigno" de la composición que tenía en men$\mathrm{te}^{26}$, hacía estudios de modelos, que posaban en su taller, con las actitudes previstas para los personajes. En el caso de la pintura del Prado, se pueden citar varios estudios para la Virgen María, como el realizado sobre un modelo desnudo (fig. 3, Uffizi, inv 11315 F) ${ }^{27}$, cuya trama, poco continua, parece responder a un sistema de proporciones y no a un cuadriculado para su posible traslación a la pintura o, más probablemente, a otro dibujo.

Bellori señala que para estudiar los pliegues de las vestimentas Barocci utilizaba mo-

25 Turner, N., Federico Barocci, París, Biro, 2001, pp. 150-151. Emiliani, 2008, pp. XLII-XLIV. Olsen, 1955, pp. 91 y ss. También FaietTi, M., “Antinomie e armonie nei disegni di Barocci” en Federico Barocci. 1535-1621. L'incanto del colore, una lezione per due secoli, Milán, 2009, pp. 76-82.

26 Término usado por el propio Barocci, según refiere Baldinucci. El "scarpigno" o "crespigno" ("scarpegn” en dialecto) es una hierba de poco valor comúnmente usada en la gastronomía de Emilia Romagna. BALdinucCI, F., Notizie de' professori del disegno (Firenze, 1681-1728), ed. BAROCCHI, P., Florencia, 1974-1975, pp. 77-78. TuRNER, 2001, p. 151, n. 7 .

${ }^{27}$ La mayoría de los dibujos relacionados con la obra se estudian en conjunto en el catálogo de Emiliani, al que nos remitimos para la abundante bibliografía al respecto. EMILIANI, 2008, pp. 188-208. 


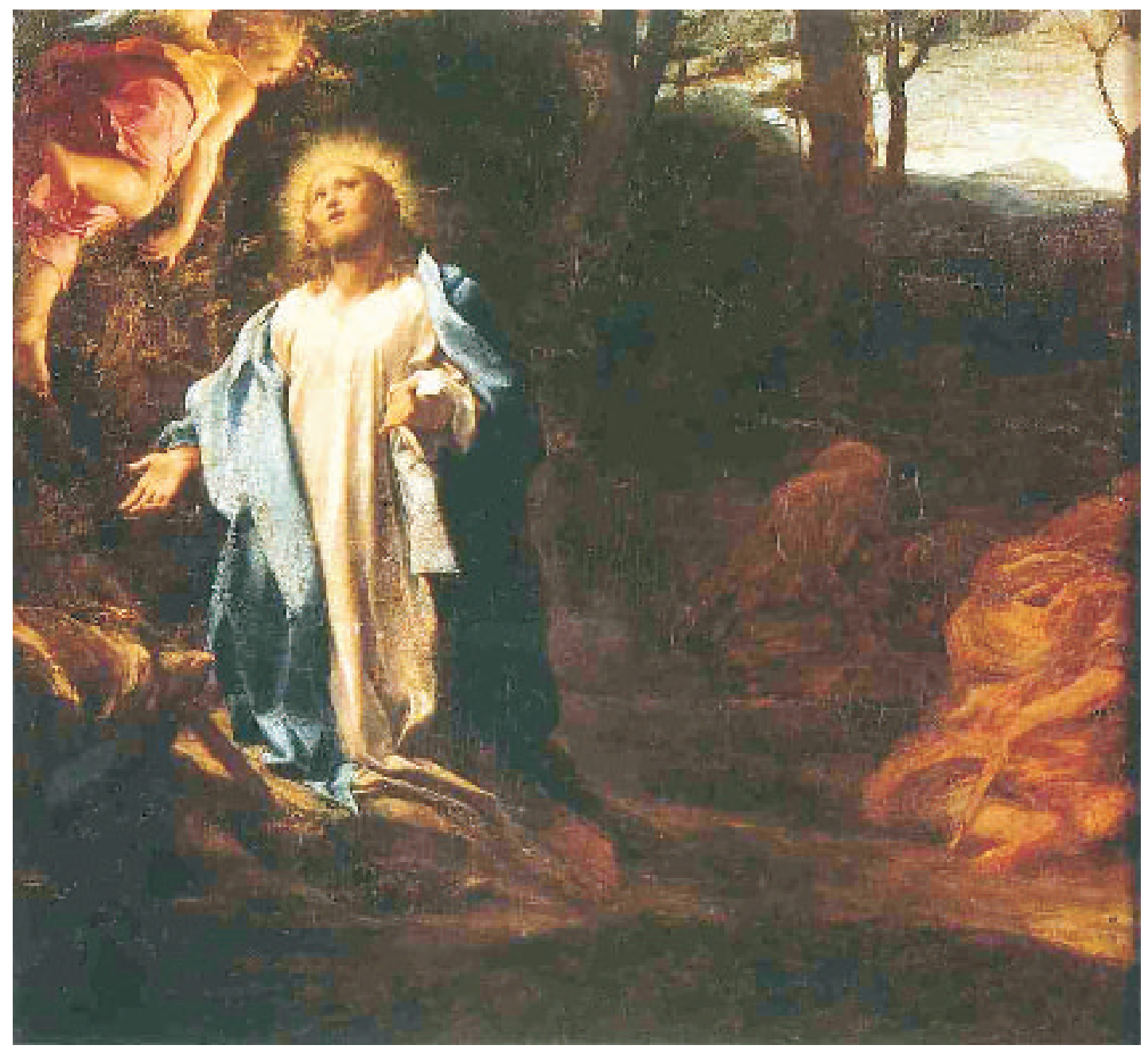

Fig. 4. Correggio. Oración en el huerto. English Heritage. Apsley House. Londres.

delos de arcilla o cera que conformaba a su gusto y que después, para evitar toda traza de artificio en su trabajo, vestía a sus modelos vivos para dibujarlos. Así, si la actitud de María deriva de la Natividad de Correggio en los Uffizi, no se ha notado que su figura se inspira claramente en la de Cristo en la Oración en el huerto de Correggio (fig. 4), ahora en Apsley House. Así lo demuestra su posición semierguida, la apertura de sus brazos y, más aún, la disposición de su pierna visible y la caída de los pesados pliegues de su túnica. Da la impresión de que Barocci hubiera modelado una estatuilla a tal efecto partiendo de la figura bidimensional de Allegri. Un pequeño modelo escultórico que habría girado hasta mostrar la posición que, invertida, adopta María en la pintura. De esta manera, Barocci intenta que su figura resulte creíble y verosímil a pesar de inspirarse abiertamente en el repertorio de otro pintor. Pretende que no resulte demasiado "alla maniera di", en ese caso, la de Correggio, en busca de ese compromiso entre artificio y naturaleza que sería tan apreciado por Bellori en su defensa del clasicismo y que le llevó a incluir a Barocci, a pesar de que éste falleció apenas iniciado el siglo, en sus Vidas de los pintores del siglo XVII. 


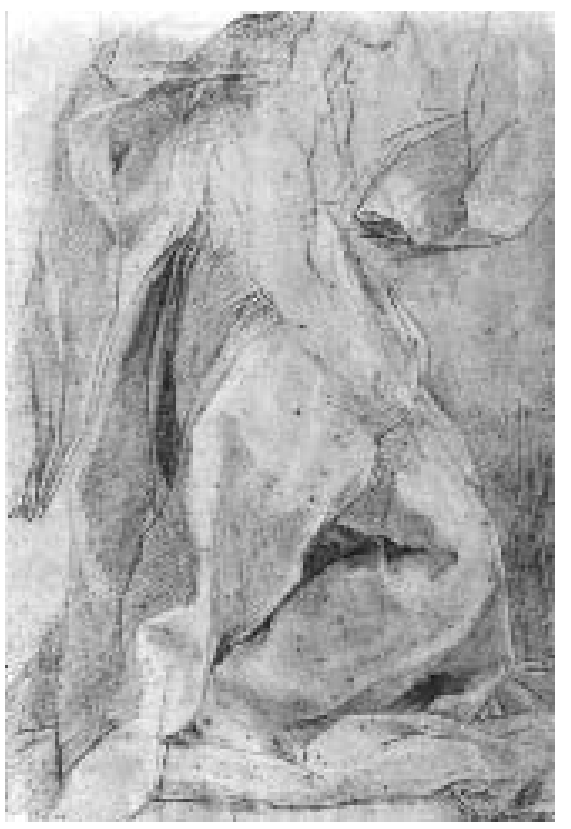

Fig. 5. Barocci. Estudio de paños. Pinacoteca Ambrosiana. Milán.

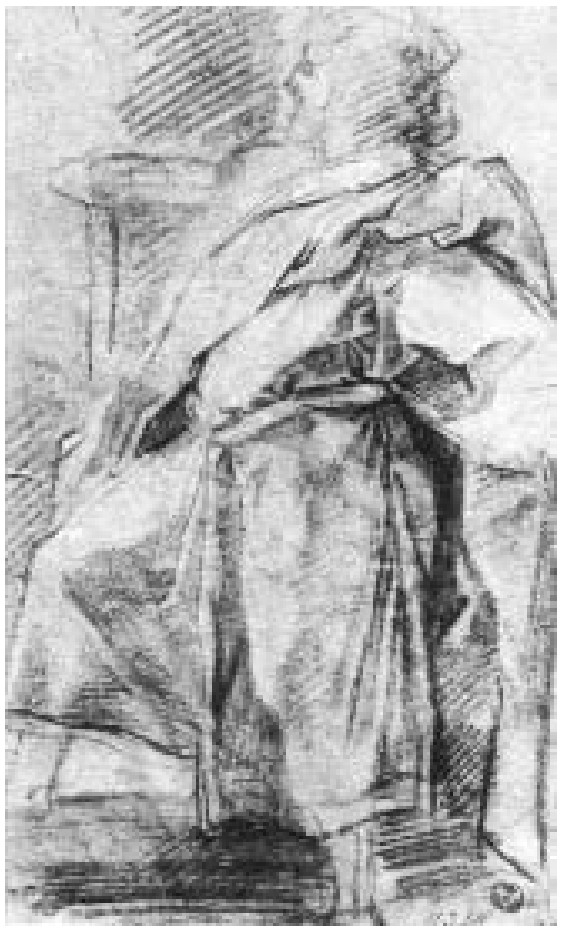

Fig. 7. Barocci. Estudio para San José. Gabinetto Disegni e Stampe degli Uffizi.

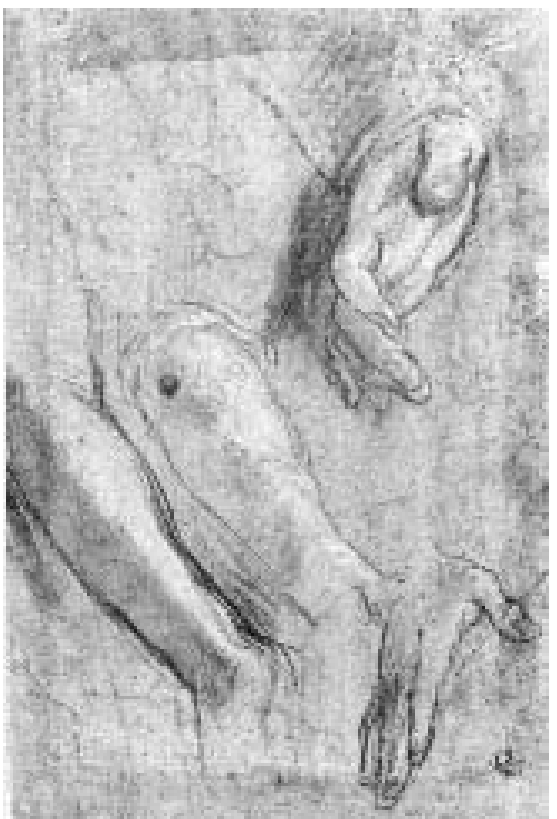

Fig. 6. Barocci. Estudio de manos. Gabinetto Disegni e Stampe degli Uffizi.

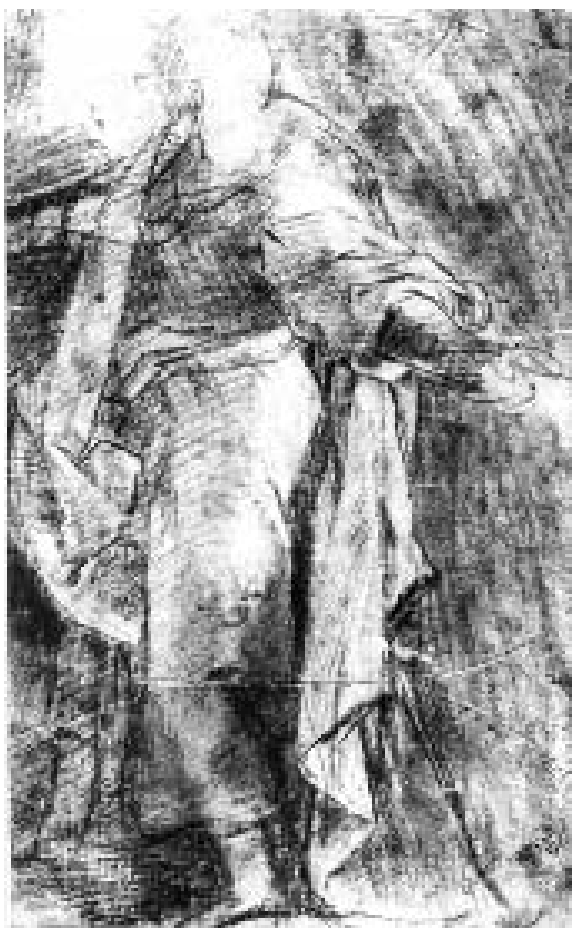

Fig. 8. Barocci. Estudio para San José. Gabinetto Disegni e Stampe degli Uffizi.

Arch. esp. arte, LXXXIV, 335, JULIO-SEPTIEMBRE 2011, 197-210, ISSN: 0004-0428 


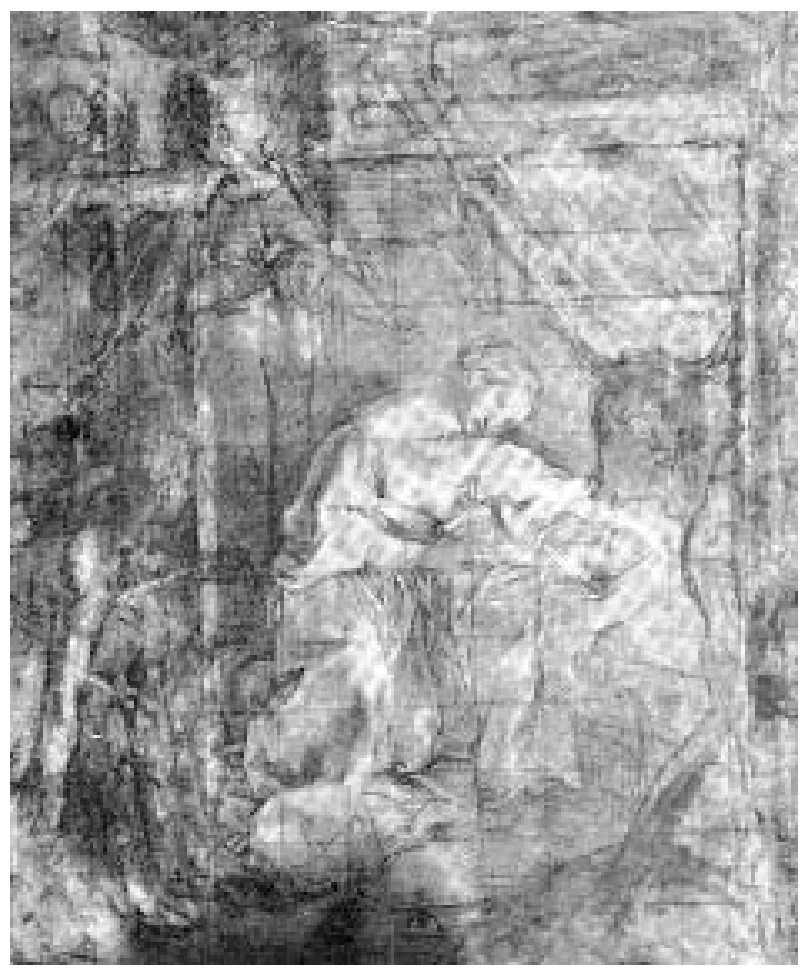

Fig. 9. Barocci. Natividad. Gabinetto Disegni e Stampe degli Uffizi.

De los estudios de paños sobre estatuillas dan fe los dibujos conservados en Milán (fíg. 5 , Inv. F. 290 Inf 2, 3 y 4), en los que los pliegues presentan un aspecto escultórico que, ciertamente, incita a pensar en la utilización de paños encolados. A estos estudios se añaden otros, en la misma fase de realización, de detalles, como los estudios de manos de los Uffizi (fig. 6, Inv 11636F r.) o Berlín (kdZ 20159 4248). También la figura de San José fue objeto de la atención de Barocci (Milán inv. 290, inf. n. 5; Uffizi. Inv 11386F, fig. 7), que lo concibió con un gesto grandilocuente que finalmente no resultó adecuado para el tono íntimo que acabó tomando la pintura, en la que utilizó un dibujo en el que el santo viste unos ropajes menos ampulosos (Uffizi. Inv 11384F, fig. 8) 28 . Este dibujo fue empleado en un "cartoncino" para una Natividad, también en los Uffizi (fig. 9, inv. 11432 F) ${ }^{29}$, lo que indica que se trata del estudio compositivo más avanzado respecto a la pintura. Anterior debe ser otro estudio general, bastante avanzado, para una Natividad que se atribuye a Barocci y se encuentra en el Départment des Arts Graphiques del Louvre (fig. 10), que no ha sido muy tenido en cuenta hasta la fecha ${ }^{30}$. En él, el espacio es mucho más profundo, y San José y los pastores quedan minimizados en el fondo, de manera que el gesto del santo no se relaciona con el grupo María y el Niño, tal y como resulta en la pintura y sucede ya en la composición de los Uffizi (fig. 9). La composición

${ }^{28}$ Idem, 2008, p. 197.

29 Olsen, 1955, p. 155. Bertelì, G. G., Disegni di Federico Barocci: catalogo della mostra, Florencia, 1975, pp. 82-83. EMiLiani, 2008, p. 189.

${ }^{30}$ Inv. $2843.34,3 \times 31,3 \mathrm{~cm}$. 


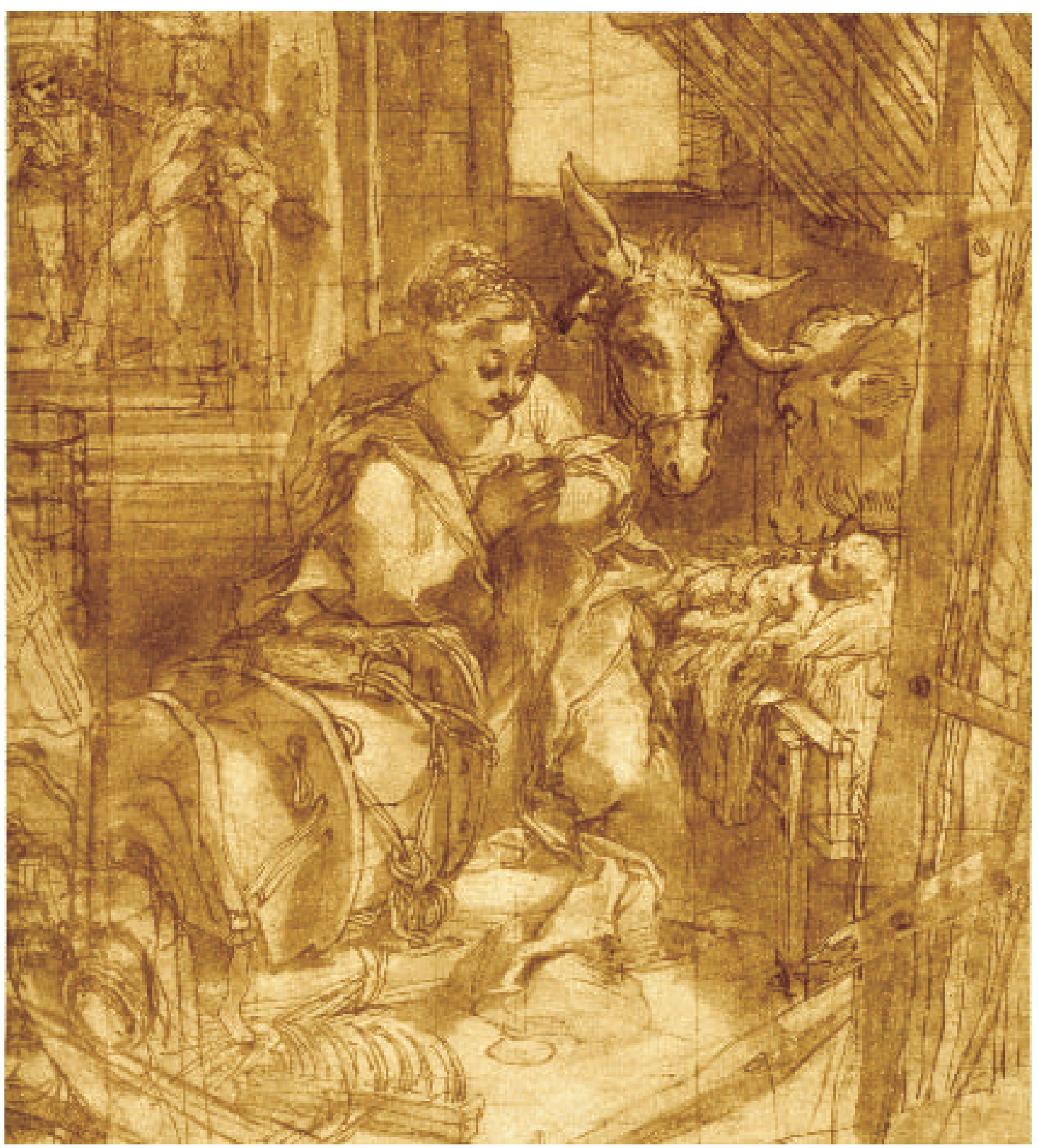

Fig. 10. Barocci. Natividad. Départment des Arts Graphiques du Musée du Louvre.

del Louvre utiliza el grandilocuente estudio para San José de los Uffizi (fig. 7), desechado en la composición final, y también un dibujo de un pastor que entra por la puerta (fig. 11 Inv. $11351 \mathrm{~F})^{31}$, lo que corrobora que probablemente se trata de una idea previa al "cartoncino" de

31 Mientras que la figura de Cristo niño se relaciona con un dibujo del Teylers Museum (Inv. E 45).

Arch. esp. arte, LXXXIV, 335, JULIO-SEPTIEMBRE 2011, 197-210, ISSN: 0004-0428 


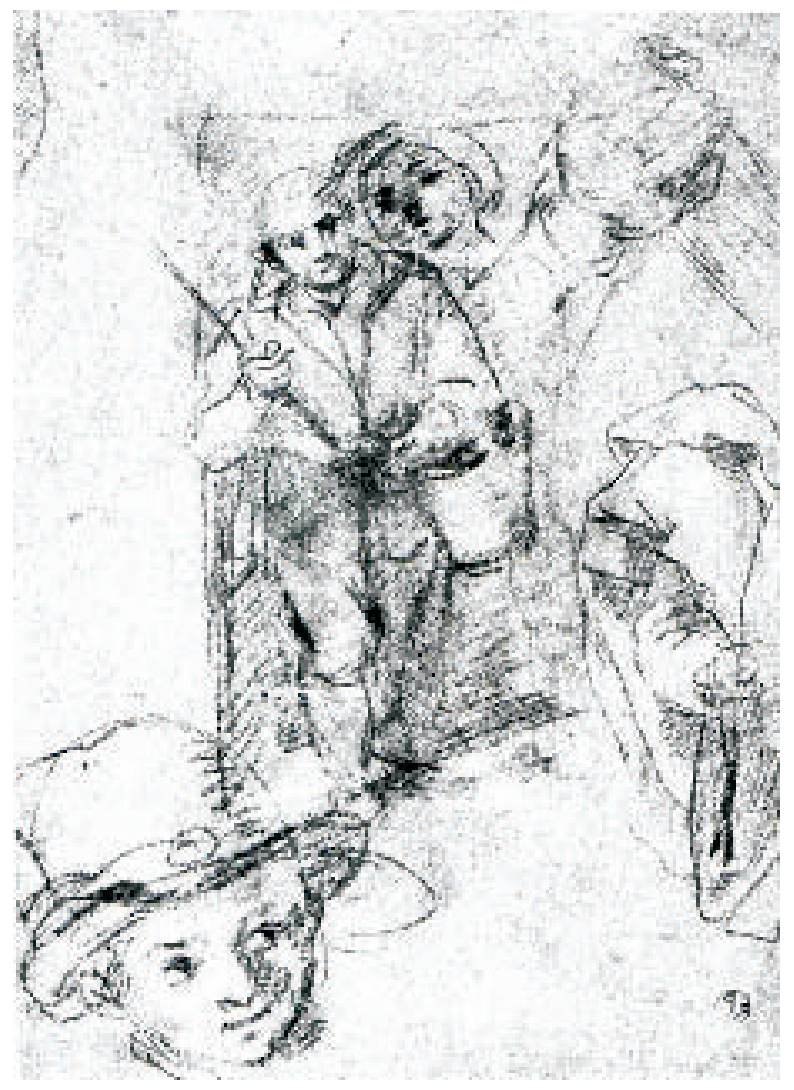

Fig. 11. Barocci. Estudio de pastor. Gabinetto Disegni e Stampe degli Uffizi. los Uffizi. Éste, junto con los estudios de María arrodillada ${ }^{32}$, demuestra que Barocci pensó en representar a la Virgen arropando al niño, inspirándose en La noche de Correggio, pero al igual que en otras obras, decidió reforzar el aspecto puramente devocional de la pintura, el "misterio", frente a su aspecto narrativo, la "istoria" ${ }^{33}$. Así, la narración queda relegada al último plano, donde los pastores son atendidos por San José, y María, por su parte, deja de arropar al niño para admirarlo, algo que ya ocurre en el dibujo del Louvre, de manera que la pintura se centra en la adoración de María al niño Jesús, recordando el cuadro del mismo tema de Allegri conservado en los Uffizi.

Cuadriculado para ser reportado sobre otra superficie es un dibujo de los Uffizi (Inv. 11150F) que representa a María y que seguramente fue trasladado al "cartonetto", para comprobar el efecto general de los diferentes estudios de figuras ordenados siguiendo una iluminación y una perspectiva común. Recientemente Verstegen y Marciari han demostrado que Barocci realizaba conjuntos de dibujos preparatorios a una misma escala, que ampliaba sucesivamente hasta llegar, a menudo trabajando al pastel, a un estudio de una mano, brazo o cabeza del tamaño que había de ocupar en el lienzo original. Como los mismos autores destacan, siempre se han interpretado estos pasteles como estudios del natural, realizados libremente, pero parece mucho más razonable considerar su ejecución cuando la pintura estaba ya avanzada, para prever su resultado final, pues a menudo son de la misma escala que el cuadro $^{34}$. Sin embargo, el extraordinario pastel para la cabeza del Niño Jesús de Windsor 5223 (fig. 12), resulta 4/3 mayor que dicha cabeza en la pintura; se ha pensado que con este aumento de escala Barocci pretendía estudiar la cabeza de manera más detallada y cuidadosa, para inyectar vida a las figuras del cuadro ${ }^{35}$, pero es posible que su mayor escala indique precisamente la intención de realizar una Natividad de mayores dimensiones que

32 The Detroit Institute of Art. William H. Murphy Fund., inv. 34139. The Fitzwilliam Museum. Cambridge, inv. 1977. Biblioteca Ambrosiana inv. F 290. Inf. N. 3. Berlin Staatliche Museen. Kupferstichkabinett inv. kdZ. 20325.

33 Como ha desarrollado brillantemente Lingo al hablar de la Madonna de la Misericordia o el Perdón de Asís. LiNGO, 2007, capítulos "Vision and Icon" e "History into Mystery", pp. 35-85.

${ }^{34}$ Marciari, J. y Verstegen, I., "Grande quanto l'opera: size and scale in Barocci's drawings", Master drawings, 46, 2008, pp. 291-321. Emiliani, 2008, p. 30.

35 Marciari, Verstegen, 2008, p. 311. Scrase. D., A Touch of the Divine: Drawings by Federico Barocci in British Collections, The Fitzwilliam Museum, Cambridge, 2006, p. 192.

Arch. esp. arte, LXXXIV, 335, JULIO-SEPTIEMBRE 2011, 197-210, ISSN: 0004-0428 


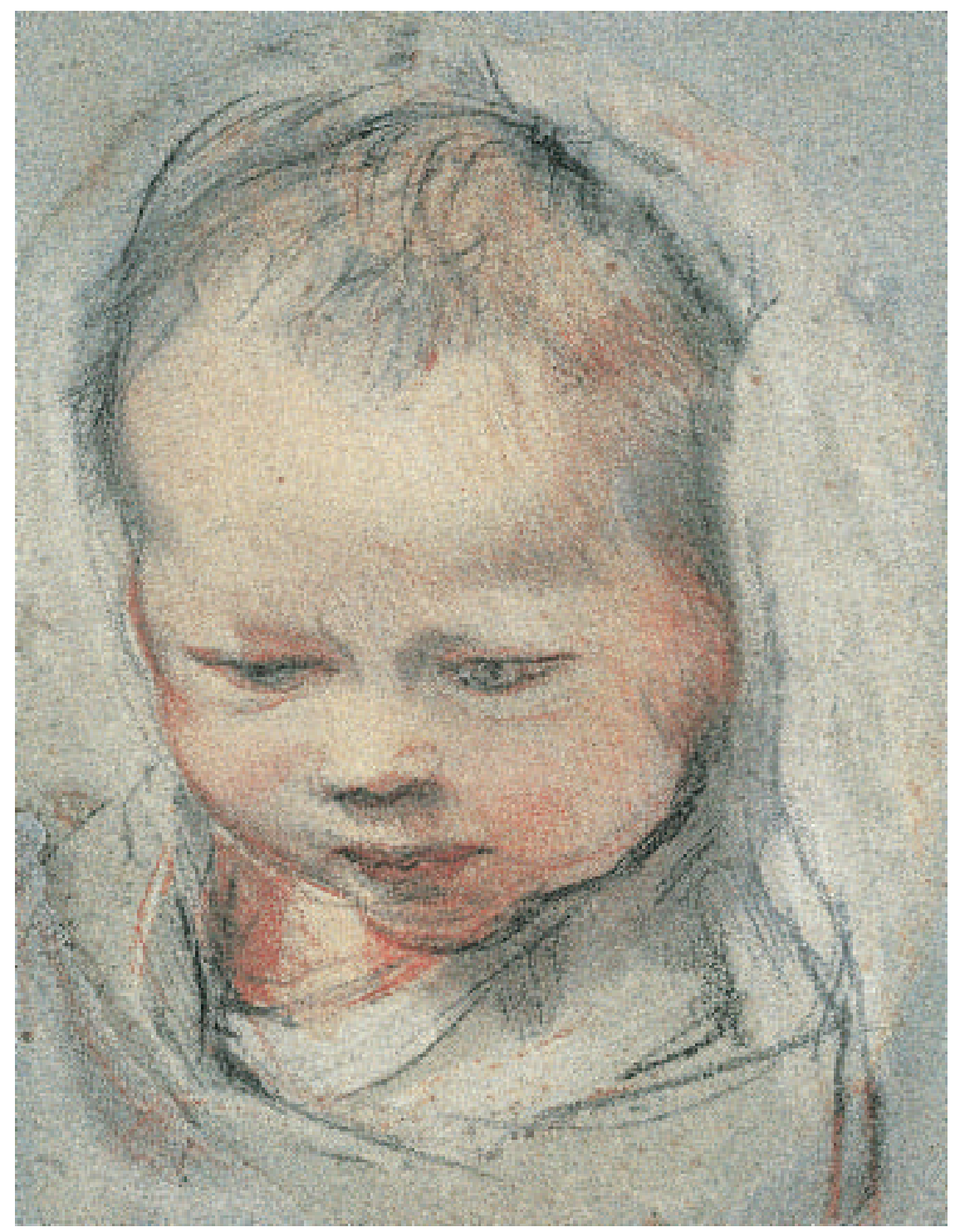

Fig. 12. Barocci. Estudio de cabeza infantil para el Niño Jesús. The Royal Collection. Windsor Castle. 


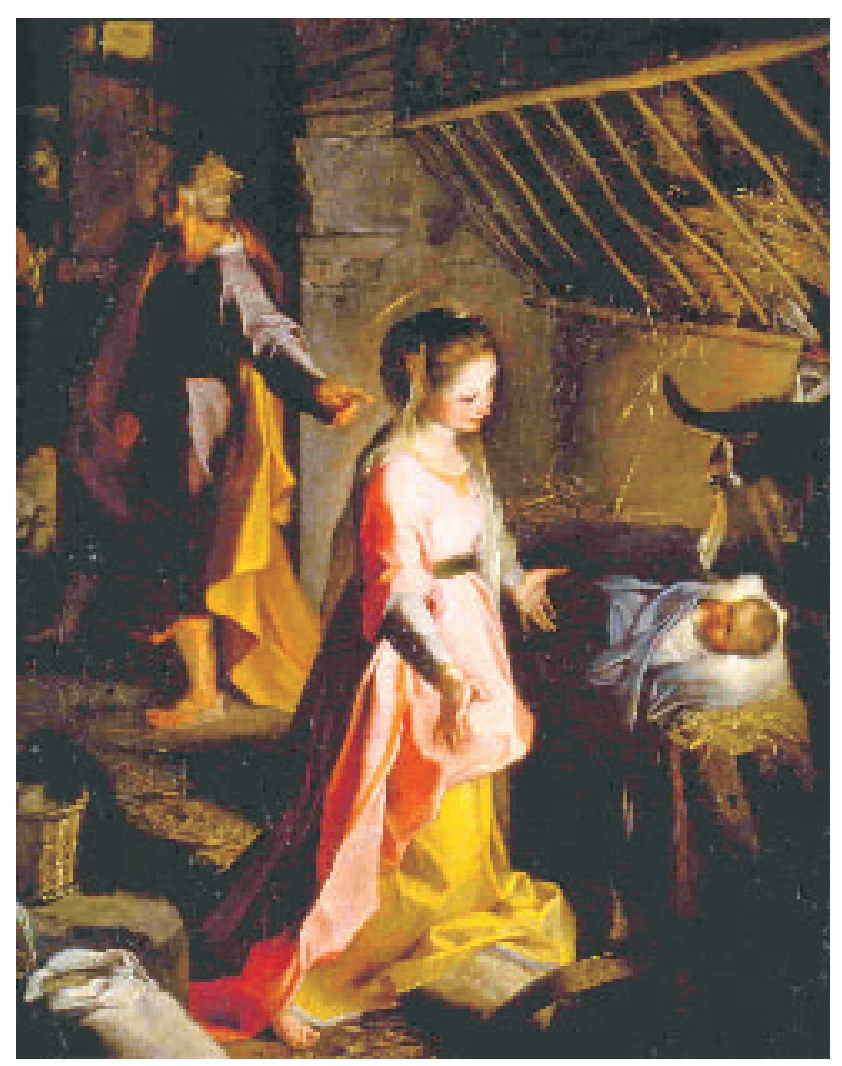

Fig. 13. Atribuido a Alessandro Vitali. Natividad. Pinacoteca Ambrosiana. Milán.

la pintura del Museo del Prado, quizá el "Presepe" encargado por el capítulo de la Catedral de Milán. De lo que no cabe duda es que la Natividad de Federico Borromeo hoy en la Ambrosiana (fig. 13) no fue pintada por Barocci, habida cuenta de la distancia existente entre la cabeza del Niño Jesús en una y otra pintura, que señala que Alessandro Vitali, al que se atribuye la copia $^{36}$, no parece haber tenido acceso al pastel mencionado.

Fecha de recepción: 22-IV-2010

Fecha de aceptación: 16-VI-2010

${ }^{36}$ A este "alter ego" de Barocci, que se caracteriza por "un leggero congelamento delle superficie cromatiche, da cui resulta una lucentezza un poco fredda" pagó Francesco Maria II, 30 escudos en 1598 por una Natividad, copiada del prototipo de Barocci, que Marchi identifica con la de Federico Borromeo. Marchi señala que probablemente el cuadro deriva del cartón original, a pesar de los cambios en los objetos de la parte derecha y la manta que cubre a Jesús. A. Marchi, “Alessandro Vitali” en Ambrosini Massari, A. M., Cellini, M., Nel segno di Barocci: allievi e seguaci tra Marche, Umbria, Siena, Milán, Motta, 2005, pp. 134-135. De la misma opinión es Emiliani. EmiLiani, 2008, II, p. 206. 